\title{
Numerical Simulation of Dam Break Flows Using a Radial Basis Function Meshless Method with Artificial Viscosity
}

\author{
Elmiloud Chaabelasri $\mathbb{D}^{1,2}$ \\ ${ }^{1}$ National School of Applied Sciences, First Mohammed University, Al-Hociema, Morocco \\ ${ }^{2}$ LME, Faculty of Sciences, First Mohammed University, Oujda, Morocco \\ Correspondence should be addressed to Elmiloud Chaabelasri; chaabelasri@gmail.com
}

Received 29 December 2017; Revised 1 March 2018; Accepted 13 March 2018; Published 15 May 2018

Academic Editor: Dimitrios E. Manolakos

Copyright (C) 2018 Elmiloud Chaabelasri. This is an open access article distributed under the Creative Commons Attribution License, which permits unrestricted use, distribution, and reproduction in any medium, provided the original work is properly cited.

A simple radial basis function (RBF) meshless method is used to solve the two-dimensional shallow water equations (SWEs) for simulation of dam break flows over irregular, frictional topography involving wetting and drying. At first, we construct the RBF interpolation corresponding to space derivative operators. Next, we obtain numerical schemes to solve the SWEs, by using the gradient of the interpolant to approximate the spatial derivative of the differential equation and a third-order explicit Runge-Kutta scheme to approximate the temporal derivative of the differential equation. For the problems involving shock or discontinuity solutions, we use an artificial viscosity for shock capturing. Then, we apply our scheme for several theoretical twodimensional numerical experiments involving dam break flows over nonuniform beds and moving wet-dry fronts over irregular bed topography. Promising results are obtained.

\section{Introduction}

Development of robust meshless methods for the numerical solution of partial differential equations has attracted considerable interest over the past twenty years, for example, [1-3]. There are three types of meshless techniques: meshless techniques based on weak forms such as the element-free Galerkin method [4], meshless techniques based on collocation techniques such as the meshless collocation technique based on radial basis functions (RBFs) [5], and meshless techniques based on the combination of weak forms and collocation technique. In the literature, several meshless weak form techniques have been reported; among others, the smooth particle hydrodynamic method [6] and boundary point interpolation method are worth noticing [7]. The weak forms are used to derive a set of algebraic equations through a numerical integration process using a set of quadrature domain that might be constructed globally or locally in the domain of the problem. In this topic, Liu et al. [8] applied the notion of meshless local Petrov-Galerkin and developed the meshless local radial point interpolation method. This method was studied and used on a class of three-dimensional wave equations later by Shivanian
[9]. A combination of the natural neighbour finite element method with the radial point interpolation method, using the multiquadric radial basis function, is developed by Dinis et al. [10] and Belinha et al. [11] to analyse a three-dimensional solid.

Initially, the radial basis function meshless method was developed for data surface fitting, and later, with the work developed by Kansa [5], the radial basis function was used for solving partial differential equations. Fedoseyev et al. [12] and Cheng et al. [13] have shown that meshless radial basis functions (RBFs) are attractive options because of the exponential convergence of certain RBFs. Various RBFs have been successfully applied to obtain accurate, efficient solutions of partial differential equations of engineering interest. This method has been applied to solve inviscid compressible flows [14], natural convection [15], heat conduction [16], three-dimensional incompressible viscous flows [17], and long waves in shallow water [18].

The Saint-Venant equations, also called shallow water equations, have often been preferred for the propagation of the flow in the open channel, and they exhibit a simplified mathematical structure, with an ability to take into account the smoothly varying flow conditions and flow discontinuities such as hydraulic jumps, moving bores, and propagation over 
dry beds, despite it still poses many theoretical and practical problems [19]. The dam break flow problem is an ideal theoretical example that involves these hydraulic challenges. In this paper, we examine the application of the radial basis function meshless method to the numerical solution of the shallow equations for dam break flow problems involving wetting/drying over complicated, frictional bed topography. In the formulation of RBFs, the radial basis functions represented by the multiquadric functions $\sqrt{1+\left(\varepsilon\left\|\mathbf{x}-\mathbf{x}_{i}\right\|\right)^{2}}$ are employed as basis functions to compute weighting coefficients for space differential operators, over a global set of computational collocation points [20]. The friction term is included in the momentum equations and discretized by a splitting implicit scheme [21]. A third-order Runge-Kutta algorithm is used for time integration [22].

It is known that the system of shallow water equations admits nonsmooth solutions that may contain shocks and rarefaction waves and, in the case of nonsmooth bottom topography, also contain contact discontinuities. To perform properly, a numerical method must be nonlinearly stable because linearly stable methods may develop large spurious oscillations and even blow up. To stabilize the proposed RBF model for slowly varying flows, as well as rapidly varying flows involving shocks or discontinuities such as dam breaks and hydraulic jumps, an artificial viscosity technique is used, following [23]. Furthermore, to avoid numerical instability caused by negative water depth near wet/dry fronts, the local flow variables are modified, imposing zero discharge when the water height became very small. As a consequence, the present numerical scheme ensures preservation of nonnegative water depth, and there is no need to limit the fluxes during simulation.

This paper is organized as follows: Section 2 outlines the shallow water equations. Section 3 presents the general formulation of partial differential problem interpolation using RBFs. Section 4 describes the application of RBFs to generate the discrete form of shallow water equations. Details are given of the numerical methods used to represent the friction term and carry out time integration. Section 4 presents the way to use the artificial viscosity for shock capturing. Section 6 discusses validation and application of the method; several numerical experiments are carried out for previously published benchmark cases in order to confirm the potential of the proposed scheme. Section 7 summarizes the main findings.

\section{Two-Dimensional Shallow Water Equations}

In conservation form, the two-dimensional nonlinear shallow water equations are given by (1) below [24]. The depth-averaged continuity and momentum equations in the $x$-and $y$-directions are

$$
\begin{gathered}
\frac{\partial h}{\partial t}+\frac{\partial(h u)}{\partial x}+\frac{\partial(h v)}{\partial y}=0 \\
\frac{\partial(h u)}{\partial t}+\frac{\partial\left(h u^{2}+\left(g h^{2} / 2\right)\right)}{\partial x}+\frac{\partial(h u v)}{\partial y}=-g h \frac{\partial z_{\mathrm{b}}}{\partial x}-\frac{S_{f x}}{\rho} \\
\frac{\partial(h v)}{\partial t}+\frac{\partial(h u v)}{\partial x}+\frac{\partial\left(h v^{2}+\left(g h^{2} / 2\right)\right)}{\partial y}=-g h \frac{\partial z_{\mathrm{b}}}{\partial y}-\frac{S_{f y}}{\rho}
\end{gathered}
$$

where $h$ is the total depth from the sea bed to the free surface, $u$ and $v$ are the depth-averaged velocity components in the Cartesian $x$ and $y$ directions, $z_{\mathrm{b}}$ is the bed elevation above a fixed horizontal datum, $g$ is the acceleration due to gravity, and $S_{f x}$ and $S_{f y}$ are the bed shear stress components, which are defined as

$$
\begin{aligned}
& S_{f x}=\rho C_{\mathrm{b}} u \sqrt{u^{2}+v^{2}}, \\
& S_{f y}=\rho C_{\mathrm{b}} v \sqrt{u^{2}+v^{2}},
\end{aligned}
$$

where $\rho$ is the water density and $C_{\mathrm{b}}$ is the bed friction coefficient, which may be estimated from $C_{\mathrm{b}}=\left(g n_{\mathrm{M}}^{2} / h^{1 / 3}\right)$, where $n_{\mathrm{M}}$ is the Manning coefficient.

$$
\frac{\partial \mathbf{W}}{\partial t}+\frac{\partial \mathbf{F}_{x}(\mathbf{W})}{\partial x}+\frac{\partial \mathbf{F}_{y}(\mathbf{W})}{\partial y}=\mathbf{S}(\mathbf{W})
$$

where $\mathbf{W}$ is the vector of dependent variables, $\mathbf{F}_{x}$ and $\mathbf{F}_{y}$ are the inviscid flux vectors, and $\mathbf{S}$ is the vector of source terms. In full, the vectors are

$$
\begin{aligned}
& \mathbf{W}=\left(\begin{array}{c}
h \\
h u \\
h v
\end{array}\right), \\
& \mathbf{F}_{x}=\left(\begin{array}{l}
h u \\
h u^{2}+\frac{g h^{2}}{2} \\
h u v
\end{array}\right), \\
& \mathbf{F}_{y}=\left(\begin{array}{l}
h v \\
h u v \\
h v^{2}+\frac{g h^{2}}{2}
\end{array}\right), \\
& \mathbf{S}=\left(\begin{array}{l}
0 \\
-g h S_{\mathrm{f} x}-g h \frac{\partial z_{\mathrm{b}}}{\partial x} \\
-g h S_{\mathrm{f} y}-g h \frac{\partial z_{\mathrm{b}}}{\partial y}
\end{array}\right) .
\end{aligned}
$$

\section{The Radial Basis Function Meshless Method}

Let $\Omega$ be an open domain of $R^{2}$. Suppose $U=U(\mathbf{x}, t)$ is a function to be approximated in a set of $N$ pairwise distinct nodes $\mathbf{x}=\left\{x_{1}, x_{2}, \ldots, x_{N}\right\}$. In the RBF meshless scheme, the approximation of $U$ at the node $\mathrm{x}=\left(x_{i}, y_{i}\right)$ can be written as a linear combination of $N$ RBFs:

$$
U(\mathbf{x}, t)=\sum_{j=1}^{N} \lambda_{j}(t) \varphi\left(\left\|\mathbf{x}-\mathbf{x}_{j}\right\|, \varepsilon\right),
$$


where $U(\mathbf{x}, t)=\left[U\left(\mathbf{x}_{1}, t\right), U\left(\mathbf{x}_{2}, t\right), \ldots, U\left(\mathbf{x}_{N}, t\right)\right]^{T}$ are the function values at node $\mathbf{x}, \Phi(\mathbf{x})=\left[\varphi\left(\left\|\mathbf{x}-\mathbf{x}_{1}\right\|, \varepsilon\right), \varphi\left(\left\|\mathbf{x}-\mathbf{x}_{2}\right\|, \varepsilon\right)\right.$, $\left.\ldots, \varphi\left(\left\|\mathbf{x}-\mathbf{x}_{N}\right\|, \varepsilon\right)\right]^{T}$ is a RBF centered at $\mathbf{x},\|$.$\| denotes the$ Euclidean norm between nodes $\mathbf{x}$ and $\mathbf{x}_{j}$, and $\Lambda=\left[\lambda_{1}, \lambda_{2}, \ldots, \lambda_{N}\right]^{T}$ are the coefficients to be determined.

One of the most commonly used RBFs is the multiquadric (MQ) RBFs [25]. Here, we use the infinitely smooth multiquadric radial basis function defined as

$$
\varphi\left(\left\|\mathbf{x}-\mathbf{x}_{j}\right\|, \varepsilon\right)=\sqrt{1+\varepsilon^{2}\left(\mathbf{x}-\mathbf{x}_{j}\right)^{2}},
$$

where $\varepsilon$ is a shape parameter that controls the fit of a smooth surface to the data. In the present work, we used the following selection [26]:

$$
\varepsilon=0.8 \frac{\sqrt{N}}{d_{\mathrm{m}}},
$$

where $d_{\mathrm{m}}$ denotes the smallest nodal distance. The expansion coefficients $\Lambda$ in (5) are obtained by solving the following linear system of $N \times N$ algebraic equations of $U\left(\mathbf{x}_{i}, t\right)=U_{i}$ :

$$
\Phi \Lambda=\mathbf{U}
$$

The expansion coefficients are then calculated by

$$
\Lambda=\Phi^{-1} \mathbf{U}
$$

where $\mathbf{U}=\left[U_{1}, U_{2}, \ldots, U_{N}\right]^{T}$ is the vector of approximate solutions and $\Phi=\left(\varphi\left(\left\|x_{i}-x_{j}\right\|, \varepsilon\right)\right), \quad 1 \leq i, j \leq N$ is an $N \times N$ matrix of RBFs given as

$$
\left(\begin{array}{ccc}
\varphi\left(\left\|x_{1}-x_{1}\right\|, \varepsilon\right) & \ldots & \varphi\left(\left\|x_{1}-x_{N}\right\|, \varepsilon\right) \\
\vdots & & \vdots \\
\varphi\left(\left\|x_{N}-x_{1}\right\|, \varepsilon\right) & \ldots & \varphi\left(\left\|x_{N}-x_{N}\right\|, \varepsilon\right)
\end{array}\right)_{N \times N} .
$$

Space derivatives of the interpolant (5) may be readily calculated, due to its linearity. Generally, the first- and second-order spatial derivatives at the point $\mathbf{x}$ can be calculated as

$$
\frac{\partial^{(k)} \mathbf{U}}{\partial x^{k}}=\sum_{j=1}^{N} \lambda_{j}(t) \frac{\partial^{(k)} \varphi}{\partial x^{k}}\left(\left\|x_{i}-x_{j}\right\|, \varepsilon\right),
$$

where $k=[1,2]$ denotes the first- and second-order derivatives. In a compact matrix form, using (9), (11) can be written as

$$
\frac{\partial^{(k)} \mathbf{U}}{\partial x^{k}}=\boldsymbol{\Phi} x^{(k)} \Lambda=\boldsymbol{\Phi} x^{(k)} \boldsymbol{\Phi} \mathbf{U}
$$

where

$$
\begin{aligned}
& \frac{\partial^{(k)} \mathbf{U}}{\partial x^{k}}=\left[\frac{\partial^{(k)} U\left(x_{1}, t\right)}{\partial x^{k}}, \frac{\partial^{(k)} U\left(x_{2}, t\right)}{\partial x^{k}}, \ldots, \frac{\partial^{(k)} U\left(x_{N}, t\right)}{\partial x^{k}}\right]^{T}, \\
& \boldsymbol{\Phi} x^{(k)}=\left[\frac{\partial^{(k)} \Phi\left(x_{1}\right)}{\partial x^{k}}, \frac{\partial^{(k)} \Phi\left(x_{2}\right)}{\partial x^{k}}, \ldots, \frac{\partial^{(k)} \Phi\left(x_{N}\right)}{\partial x^{k}}\right]^{T} .
\end{aligned}
$$

\section{Discretized Form of Shallow Water Equations by the RBF Meshless Method}

4. 1. Convective Flux and Bottom Topography Term Approximations. Let us assume that $F_{\xi}^{n}(\mathbf{W}(x))$ are known convective fluxes along the axes $x$ and $y$ at time $t=n \Delta t$, where $\xi=(x, y)$. Using (5), it can be approximated by

$$
F_{\xi}^{n}(W)=\sum_{j=1}^{N} \lambda_{\xi, j}(t) \varphi\left(\left\|x-x_{j}\right\|, \varepsilon\right) .
$$

In the matrix form, this equation becomes

$$
F_{\xi}^{n}=\Lambda_{\xi}^{n} \Phi \text {. }
$$

However, the expansion coefficients are then calculated by

$$
\Lambda_{\xi}^{n}=\Phi^{-1} F_{\xi}^{n}
$$

From (14), the partial flux derivative $\left(\partial F_{\xi}^{n}(W) / \partial \xi\right)$ can be written as

$$
\frac{\partial F_{\xi}^{n}(\mathbf{W})}{\partial \xi}=\sum_{j=1}^{N} \lambda_{\xi, j}^{n}(t) \frac{\partial \varphi\left(\left\|\mathbf{x}-\mathbf{x}_{j}\right\|, \varepsilon\right)}{\partial \xi} .
$$

Then, the compact matrix form of the partial derivative of the flux vector is

$$
\frac{\partial F_{\xi}^{n}(\mathbf{W})}{\partial \xi}=\Phi_{\xi} \Lambda_{\xi}^{n}=\Phi_{\xi} \Phi^{-1} F_{\xi}^{n}(\mathbf{W})
$$

Applying the same procedure described above for the bottom topography function $z_{\mathrm{b}}(\mathbf{x})$, it is easy to obtain its discrete form. The topography function can be approximated by

$$
z_{b}^{n}(\mathbf{x})=\sum_{j=1}^{N} \alpha_{j}(t) \varphi\left(\left\|\mathbf{x}-\mathbf{x}_{j}\right\|, \varepsilon\right) .
$$

Its partial derivative is then expressed as

$$
\frac{\partial z_{b}^{n}(\mathbf{x})}{\partial \xi}=\sum_{j=1}^{N} \alpha_{j}(t) \frac{\partial \varphi\left(\left\|\mathbf{x}-\mathbf{x}_{j}\right\|, \varepsilon\right)}{\partial \xi} .
$$

In the same way, the compact matrix form of the bottom topography term $S_{z_{b}}^{n}$ along the axis $\xi$ is given as

$$
S_{z_{b}}^{n}(\xi)=-g h^{n} \frac{\partial z_{b}^{n}(x, t)}{\partial \xi}=-g h^{n} \Phi_{\xi} \Phi^{-1} z_{b}^{n}(x, t) .
$$

4.2. Addition of Friction Effects. To incorporate friction into the present numerical scheme, the friction term is discretized using an operator splitting procedure described by Boushaba et al. [27], which splits the shallow water equations (2) into two equations:

$$
\begin{aligned}
& \frac{\partial h \mathbf{U}}{\partial t}=S_{b}^{n+1}(\mathbf{W})=\left(-g n^{2}\|\mathbf{U}\| \mathbf{U} h^{-1 / 3}\right)^{n+1}, \\
& \frac{\partial h \mathbf{U}}{\partial t}+\mathbf{L}\left(\mathbf{W}, Z_{\mathrm{b}}\right)=0
\end{aligned}
$$




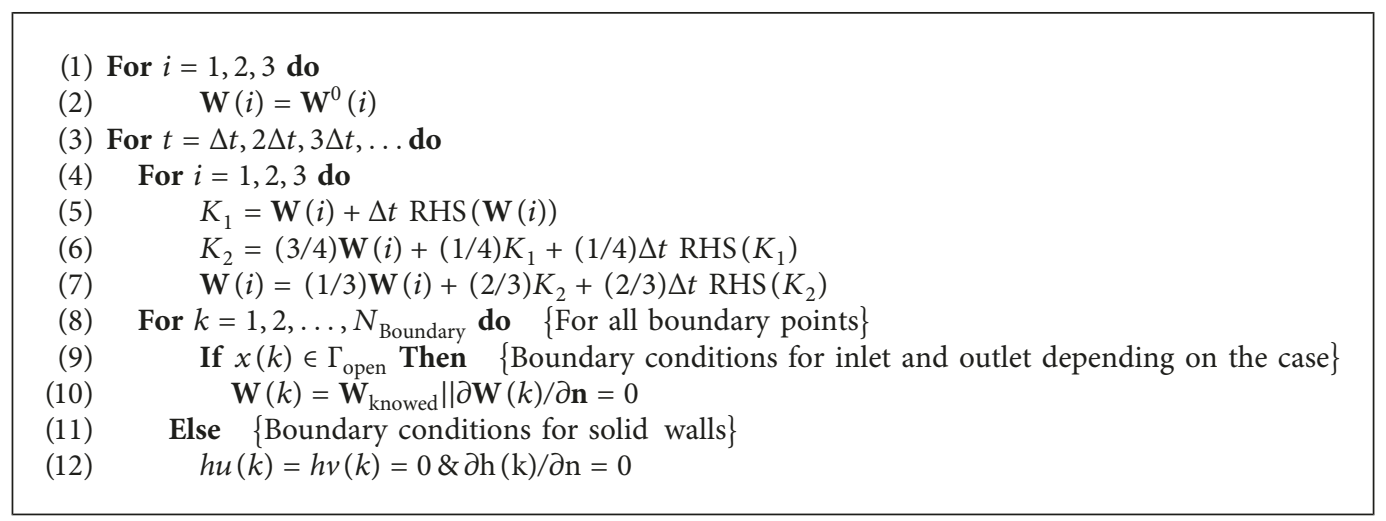

Algorithm 1: Time integration procedure.

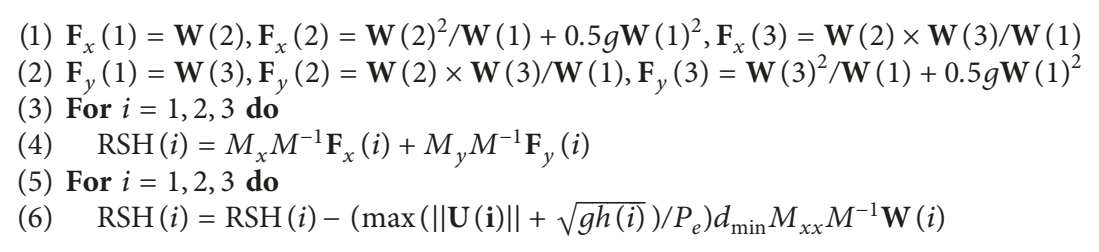

Algorithm 2: Calculation of flux vector and right-hand side.

where $U=(u, v)$. In the first step of the calculation, the upper ordinary differential equation in (23) is approximated by an implicit method as described in [21]:

$$
\frac{\overline{h \mathbf{U}}-(h \mathbf{U})^{n}}{\Delta t}=S_{b}^{n+1}(\mathrm{~W}) \text {, }
$$

where the friction term $S_{b}^{n+1}$ may be expressed using a Taylor series as

$$
S_{\mathrm{b}}^{n+1}(\mathbf{W})=S_{\mathrm{b}}^{n}(\mathbf{W})+\left(\frac{\partial S_{\mathrm{b}}}{\partial h \mathbf{U}}\right)^{n}\left((\overline{h \mathbf{U}})-(h \mathbf{U})^{n}+0\left(\Delta(h \mathbf{U})^{2}\right) .\right.
$$

Rearranging the above equation leads to the following formula for updating water discharge $\overline{h u}$ at the new time step:

$$
\overline{h \mathbf{U}}=(h \mathbf{U})^{n}+\Delta t \frac{S_{b}^{n}(\mathbf{W})}{1+2 \Delta t C_{\mathrm{f}}\|\mathbf{U}\| h^{-1}} .
$$

In the second step, the value $\overline{h \mathbf{U}}$ is taken to be the initial condition when solving the second equation in (23).

4.3. Time Integration and Stability Condition. To date, the forward Euler method has been mainly used as the preferred time-stepping scheme for RBF methods. However, the forward Euler method is only the first-order accurate in time and so may introduce excessive numerical dissipation in the computed RBF solutions. To achieve a higher order of accuracy, we use the explicit Runge-Kutta method recommended by [22]. The procedure to advance the solution from the time $t^{n}$ to the next time $t^{n+1}$ is carried out as

$$
\begin{aligned}
\mathbf{W}^{(1)} & =\mathbf{W}^{n}+\Delta t \mathbf{L}\left(\mathbf{W}^{n}\right) \\
\mathbf{W}^{(2)} & =\frac{3}{4} \mathbf{W}^{n}+\frac{1}{4} \mathbf{W}^{(1)}+\frac{1}{4} \Delta t \mathbf{L}\left(\mathbf{W}^{(1)}\right), \\
\mathbf{W}^{n+1} & =\frac{1}{3} \mathbf{W}^{n}+\frac{2}{3} \mathbf{W}^{(2)}+\frac{2}{3} \Delta t \mathbf{L}\left(\mathbf{W}^{(2)}\right),
\end{aligned}
$$

where $n$ represents the time level and $\Delta t$ is the time step. To achieve stability, for this explicit scheme, the time step must meet the following criterion:

$$
\Delta t=\mathrm{CFL} \frac{d_{\min }}{\max \left(\left\|u_{i}\right\|+\sqrt{g h_{i}}\right)},
$$

where CFL is the Courant number, such that $0<\mathrm{CFL}<1$, and $d_{\min }$ denotes the smallest nodal distance between collocation points.

4.4. Boundary Conditions. In this work, transmissive boundary conditions for open inflow/outflow and reflective boundary conditions for solid walls are applied in the simulations. At a transmissive boundary, the flow variables at a collocation point on the boundary are set to the same values as at the interior point normal to the boundary. At a reflective boundary, the value at a collocation point is simply the mirror image of that at the associated boundary point so that the normal velocity component is zero at the boundary. However, the representation of boundary conditions within an RBF method is less trivial.

4.5. Implementation Algorithms. For a given initial condition $\mathbf{W}^{0}=\mathbf{W}(\mathbf{X}, t=0)$, the time integration procedure is 


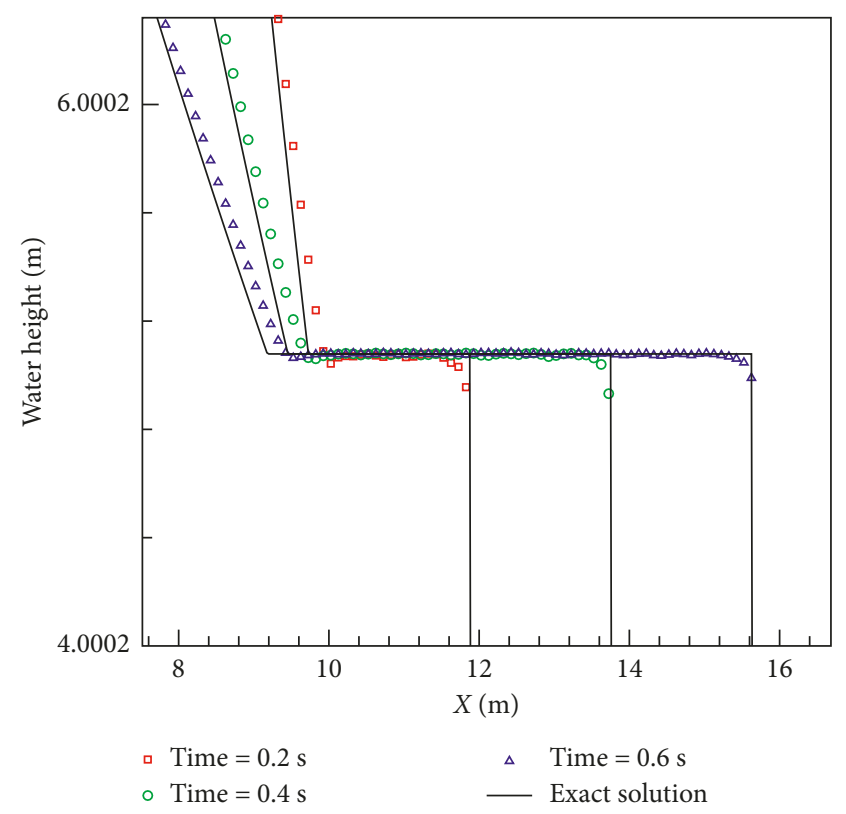

(a)

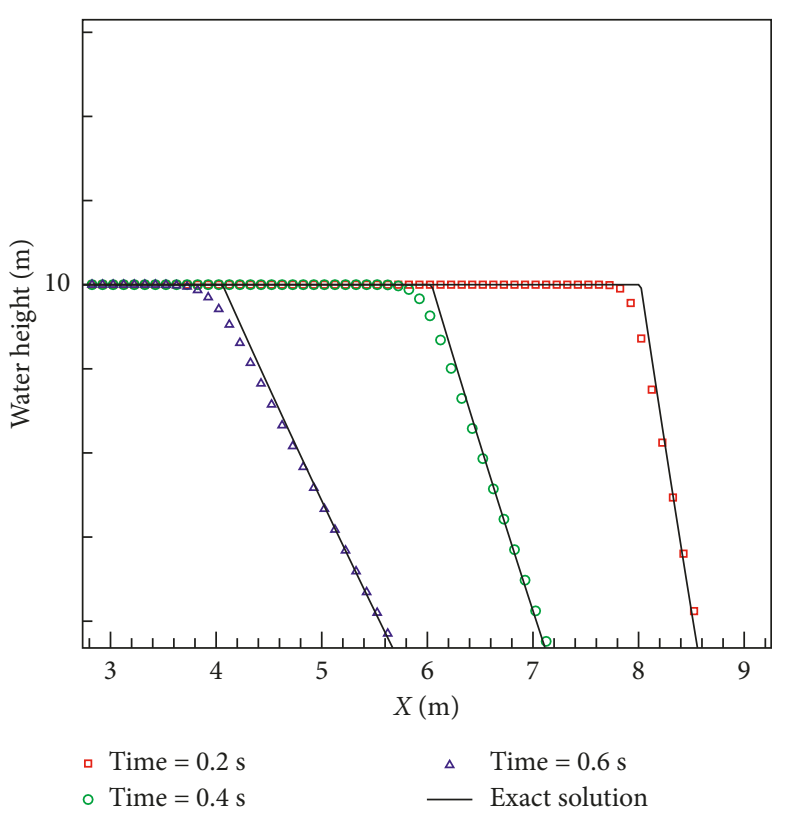

(b)

Figure 1: Numerical and exact solutions of a dam break on a wet bed, at different times, using a grid of 800 points: (a) free surface elevation profiles; (b) profiles of water discharge per unit breadth.

described in Algorithm 1. The right-hand side (RHS) used in the algorithm represents the convective flux and bottom topography that are calculated in Algorithm 2.

\section{Artificial Viscosity for Shock Capturing}

For particular hydraulic problems involving shock waves, such as a hydraulic jump or dam break flows, the numerical model is required to represent a steady or unsteady discontinuity, where the presence of oscillations in the solution is expected, and it sometimes may grow over time. However, by introducing a small amount of artificial diffusion, it is possible to damp these oscillations $[23,28]$. We therefore augment the right-hand side of the hyperbolic system (2) with the artificial diffusion term:

$$
\mathbf{D}(\mathbf{W})=D_{\mathrm{h}} \frac{\partial^{2} \mathbf{W}}{\partial x^{2}},
$$

where $D_{\mathrm{h}}$ is a tunable viscosity coefficient. The Peclet number is defined as follows:

$$
P_{e}=\frac{\max \left(\left\|\mathbf{U}_{i}\right\|+\sqrt{g h_{i}}\right)}{\mathbf{D}_{\mathbf{h}}} \mathbf{d}_{\min } .
$$

It often controls the stability of the numerical solutions $[23,29]$. In the case of convection-dominated flow, the Peclet number is large but finite, and the effect of the diffusion term (29) becomes negligible. Therefore, a natural and simple way to stabilize the solution is to reduce the Peclet number.

Using the RBF interpolation of an arbitrary function (12), the augmented term (29) can be obtained as

$$
\mathbf{D}(\mathbf{W})=\frac{\max \left(\left\|\mathbf{U}_{i}\right\|+\sqrt{g h_{i}}\right)}{P_{e}} d_{\min } \Phi_{x x} \Phi \mathbf{W}
$$

\section{Numerical Results}

6.1. One-Dimensional Dam Break over a Wet Bed. A onedimensional dam break over a wet, horizontal bed is first simulated to demonstrate the shock-capturing capability of the corrected radial basis function method using artificial viscosity. The channel is of length $20 \mathrm{~m}$. The initial water discharge and depth are given by

$$
h u(x, 0)=0 \text { and } h(x, 0)= \begin{cases}10, & \text { if } x \leq 10, \\ 2, & \text { otherwise }\end{cases}
$$

As boundary conditions, a zero discharge and a free boundary are considered at the left and right ends of the channel. The analytical solution for this simple dam break test consists of a backward-propagating rarefaction and a forward-moving shock wave. Figure 1 shows the numerical results of flow after the dam fails, at three different times in terms of water surface elevation and discharge. Here, the model domain consists of 800 points. An artificial viscosity of $0.001 \mathrm{~m}^{2} \mathrm{~s}^{-1}$ is applied corresponding to a Peclet number of 300. Figure 2 shows close-up views of the evolving free surface elevation and discharge profiles along the channel at different times. In general, satisfactory agreement is achieved between the numerical and analytical solutions, although a very small amount of numerical diffusion occurs where the surface gradient is steepest. 


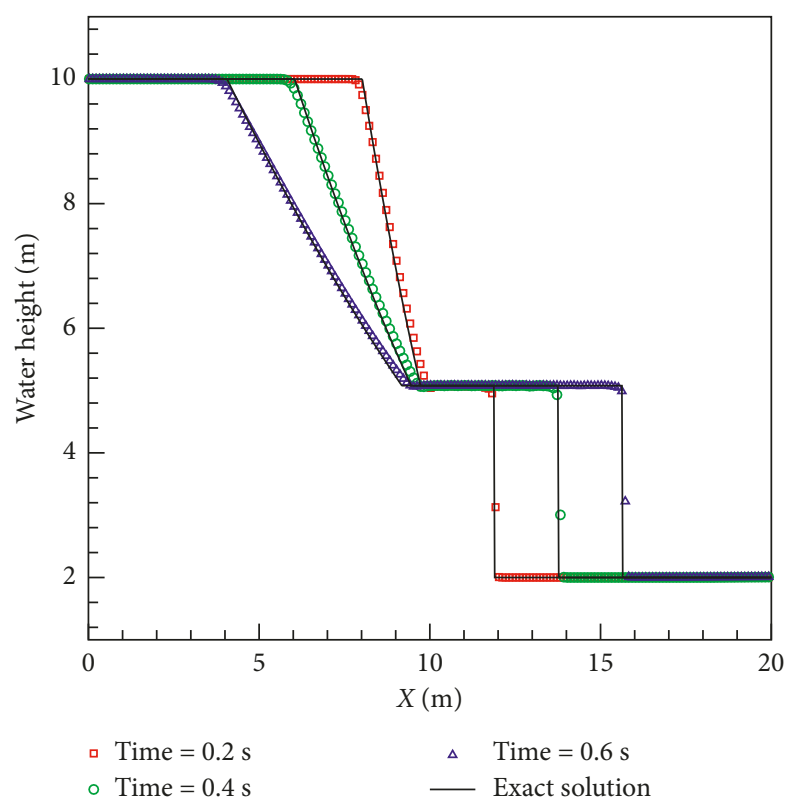

(a)

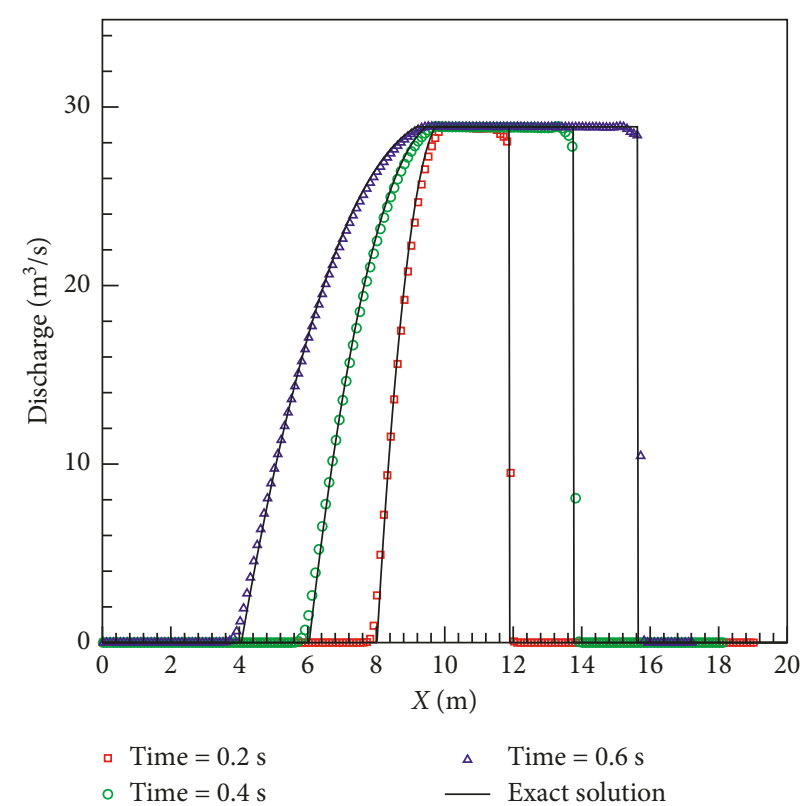

(b)

Figure 2: Zoom-in view of Figure 1, focusing on discontinuities in the water level.

6.1. Two-Dimensional Partial Dam Break Simulation. For this second test case, we consider a hypothetical twodimensional dam break problem with nonsymmetric breach that is a typical validation made in many presented papers, for example, [30-33]. An illustration of this problem is shown in Figure 3, in which the domain is defined by a $200 \mathrm{~m} \times 200 \mathrm{~m}$ channel with the horizontal bed. A dam is located in the middle of the domain, and the nonsymmetric breach is $75 \mathrm{~m}$ wide with negligible thickness and is located $95 \mathrm{~m}$ from the left side of the domain. The initial water discharge and depth are given by

$$
\begin{aligned}
& h u(t=0, x, y)=h v(t=0, x, y)=0, \\
& h(t=0, x, y)= \begin{cases}10, & \text { if } x \leq 95, \\
5, & \text { otherwise. }\end{cases}
\end{aligned}
$$

As boundary conditions, at the left and right ends of the channel, a zero discharge and a free boundary are considered, and a solid wall is considered for others. Figure 4 shows the numerical results of flow after the dam fails, at three different times in terms of water depth and velocity. As we can show, the upstream water is released into the downstream side through the breach, creating the surge wave propagating to downstream and the neglective wave to upstream. Here, the model domain consists of 2000 points. An artificial viscosity of $0.001 \mathrm{~m}^{2} \mathrm{~s}^{-1}$ is applied corresponding to a Peclet number of 300 . No analytical solution is available for this case, but the results can be compared with those of other numerical schemes. Generally, the results are in agreement with the results obtained by other numerical methods in aforementioned literatures.

6.2. Circular Dam Break. This second test case consists of the instantaneous breaking of a cylindrical tank of diameter

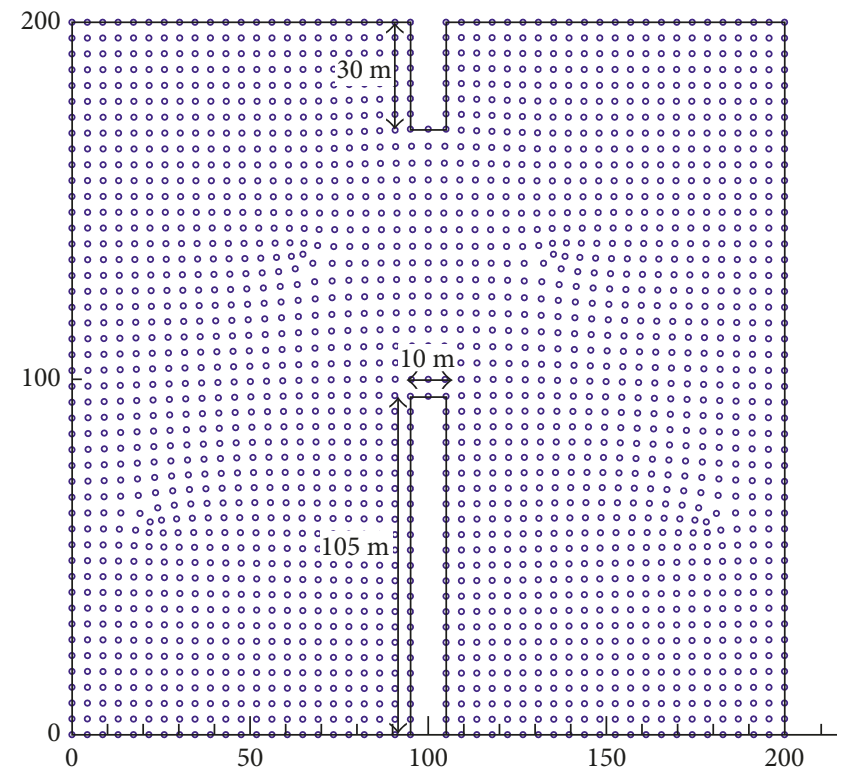

FIgURE 3: The computational domain and RBF collocation points of the $2 \mathrm{D}$ dam break problem.

$20 \mathrm{~m}$ (Figure 5), initially filled with $2 \mathrm{~m}$ of water at rest. The wave generated by the breaking of the tank propagates into still water with an initial depth of $0.5 \mathrm{~m}$. This classic problem is widely used to test the shock capture capability of numerical schemes $[34,35]$.

Figure 6 illustrates the wave propagation on computational 8000 collocation points. An artificial viscosity of $0.04 \mathrm{~m}^{2} \mathrm{~s}^{-1}$ is applied corresponding to a Peclet number of 100. At the beginning, that is, at $t=0 \mathrm{~s}$, the dam is broken instantaneously and the column of water is released, and the 

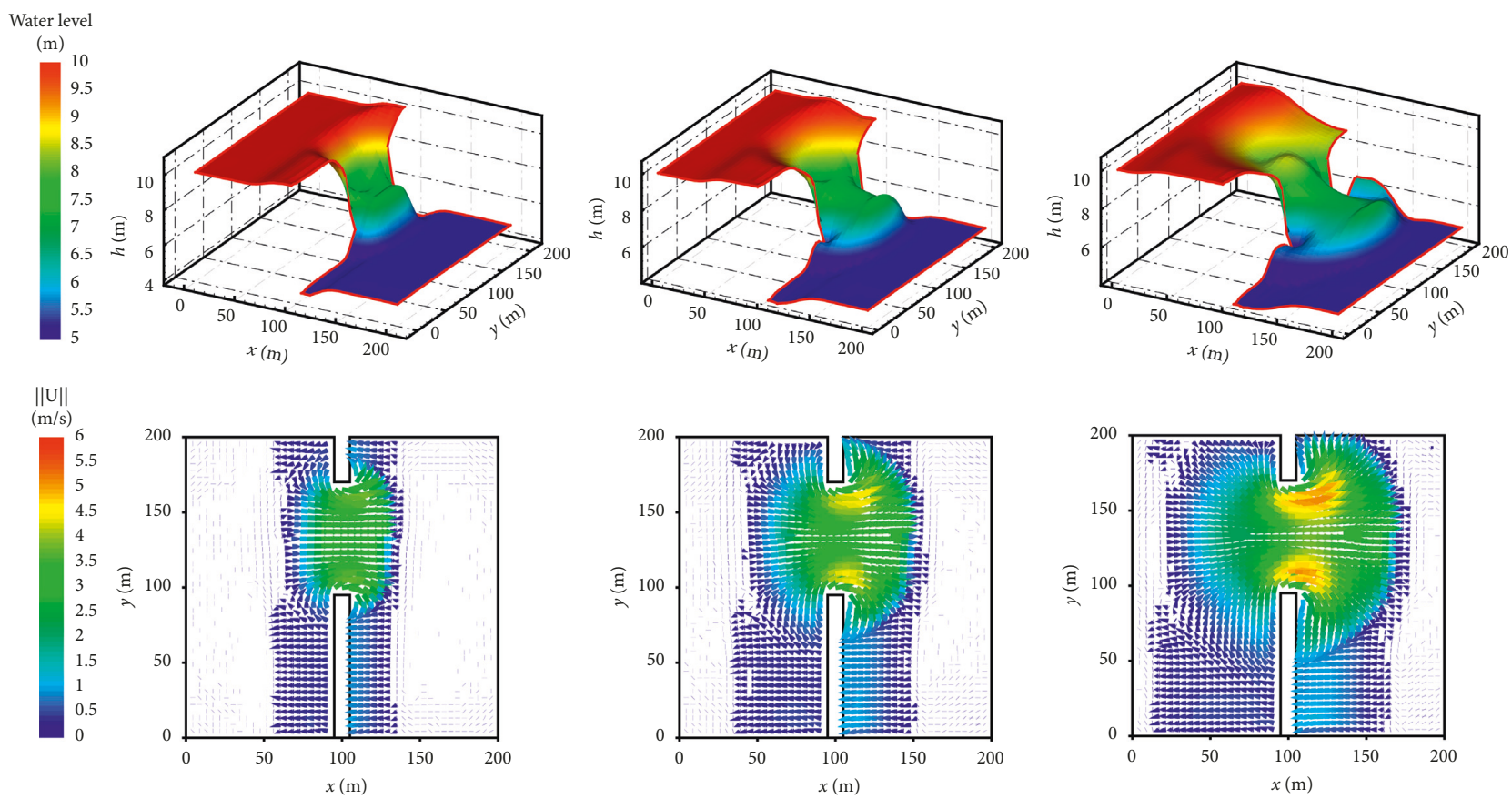

FIGURE 4: The profile of water depth and velocity vectors of the $2 \mathrm{D}$ dam break at different output times: $t=3 \mathrm{~s}, t=5 \mathrm{~s}$, and $t=7.2 \mathrm{~s}$.

shock wave results in the increase of water depth in the lower depth region, propagating in the radial direction. Our state solutions obtained at time $t=1$ and $2.5 \mathrm{~s}$ by different methods, that is, the RBF meshless method and the triangular finite volume method, as a based mesh method and a 1D reference solution are in an agreement (Figure 7). No singular corner effects on smoothness of the solution can be noticed.

6.3. Dam Break over Three Humps. The nonlinear shallow water equations are not too many analytical solutions for the problem of the flow over wetting and drying topography. In this context, Antuono et al. [36] studied an analytical solution of the shallow water flow over uneven topography, and this is for analysis of wave run-up on a beach. The current shallow water model is applied to simulate a dam break over an initially dry floodplain with three humps, recommended by Kawahara and Umetsu [37] for this challenging problem that involves complex flow hydrodynamics, bottom topography, and wetting and drying. The simulated setup is sketched in Figure 8, where the dam break occurs in a $75 \times 30 \mathrm{~m}$ rectangular domain with the dam located $16 \mathrm{~m}$ away from the upstream end. A reservoir with a still water surface elevation of $1.875 \mathrm{~m}$ is assumed to be upstream of the dam. The bed topography of the domain is defined as

$$
\begin{aligned}
z(x, y)= & \max \left[0,1-\frac{1}{8} \sqrt{(x-30)^{2}+(y-6)^{2}},\right. \\
& 1-\frac{1}{8} \sqrt{(x-30)^{2}+(y-24)^{2}}, \\
& \left.1-\frac{3}{10} \sqrt{(x-47.5)^{2}+(y-15)^{2}}\right] .
\end{aligned}
$$

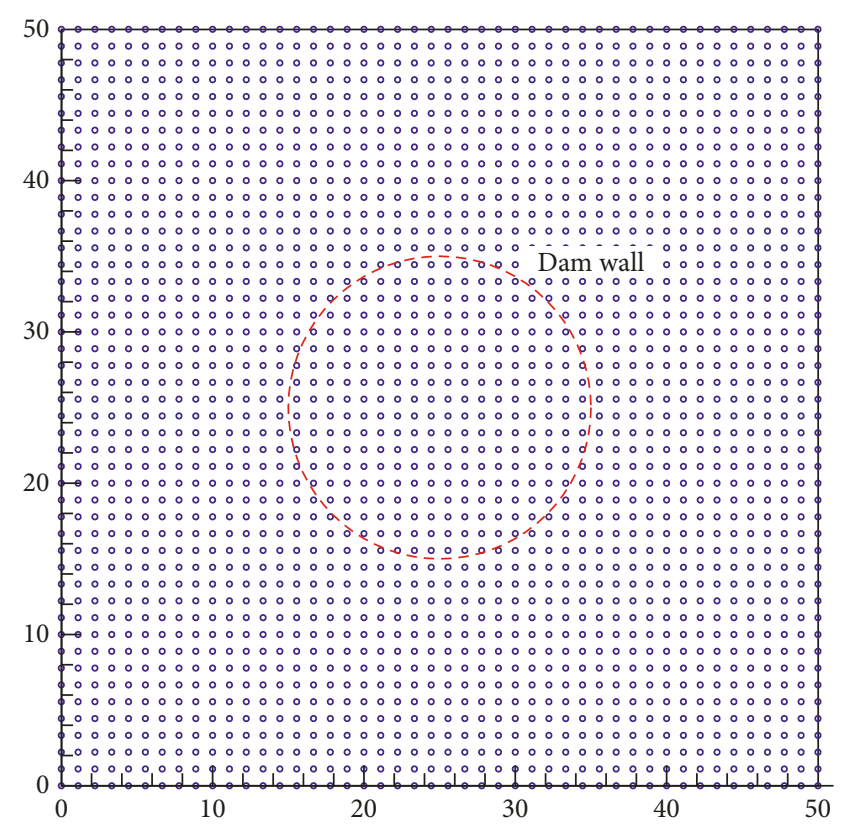

FIgURE 5: The computational domain, RBF collocation points, and location of the circular dam break problem.

The floodplain is initially dry, and a constant Manning coefficient of $0.018 \mathrm{~s} / \mathrm{m}^{1 / 3}$ is used throughout the domain. The domain walls are assumed to be solid. The dam collapses instantly at $t=0 \mathrm{~s}$, and simulation is run for $300 \mathrm{~s}$ on 2000 collocation points.

After the dam fails, the initial still water in the reservoir rushes onto the downstream floodplain. In Figure 9, we present the profile of water depth and contours at different 

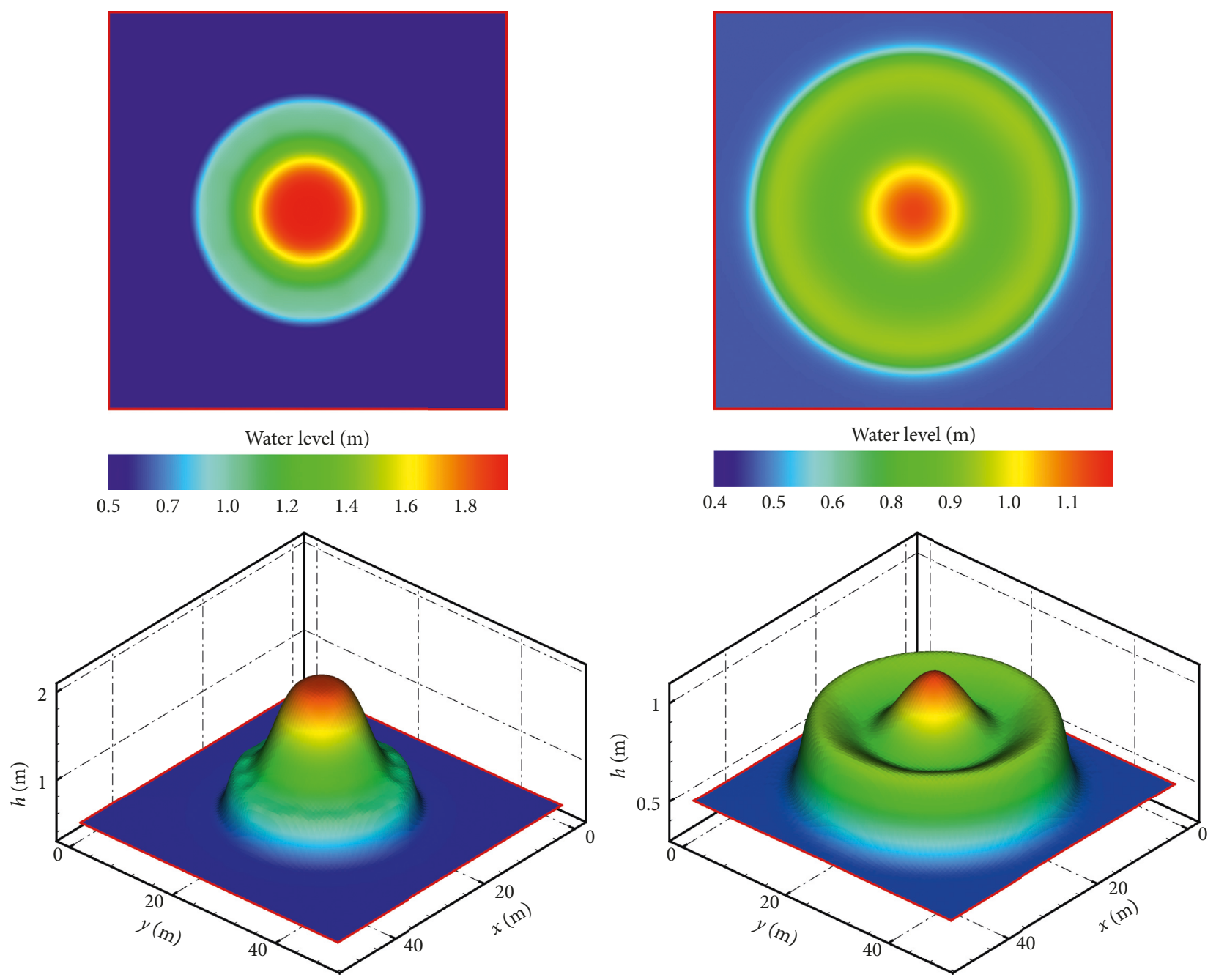

Figure 6: The water depth contours and profile of the circular dam break at different output times: $t=1 \mathrm{~s}$ and $t=2.5 \mathrm{~s}$.

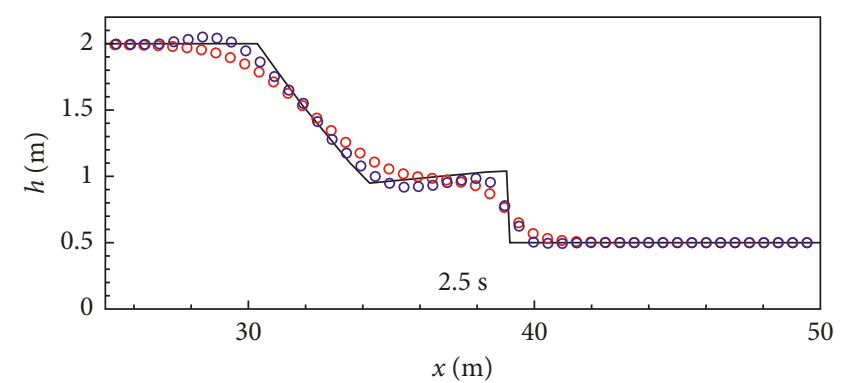

- 1 reference solution (Canestrelli et al. [35])
RBF meshless (8000 points)
$\circ \quad$ 2D-FV-ROE (8000 triangles)

(a)

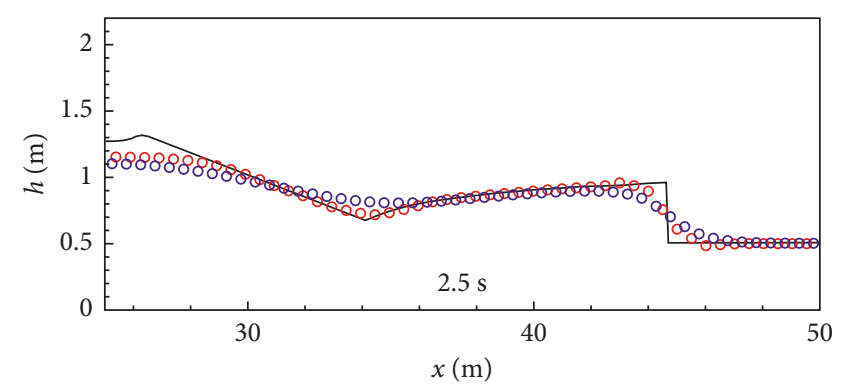

- 1D reference solution (Canestrelli et al. [35])

- RBF meshless (8000 points)

- 2D-FV-ROE (8000 triangles)

(b)

FIGURE 7: Cross section comparison between the numerical solution using the RBF meshless method and 2D FV-Roe scheme and the 1D reference solution.

output times $t=1,6,12,30$, and $300 \mathrm{~s}$. As can be observed from these results, after about $1 \mathrm{~s}$, the wet-dry front reaches the two small humps and begins to climb over them. At $t=6.0 \mathrm{~s}$, the two small humps are entirely submerged and the wave front hits the large hump. At $t=12 \mathrm{~s}$, the wave front passes through both sides of the large hump and begins to submerge the lee of the hump. Finally, the flow becomes steady due to the dissipation caused by bed friction, as shown at $t=300 \mathrm{~s}$ when the flow is nearly motionless and the peaks of the humps are no longer submerged. 


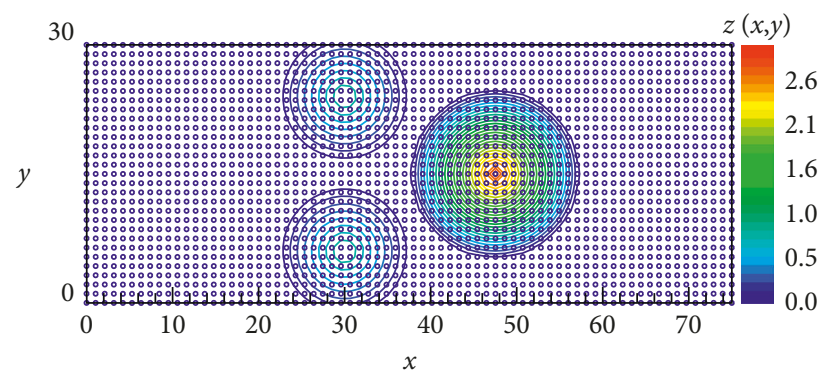

FIGURE 8: The computational domain, RBF collocation points, and bed contours of the dam break problem over humps.
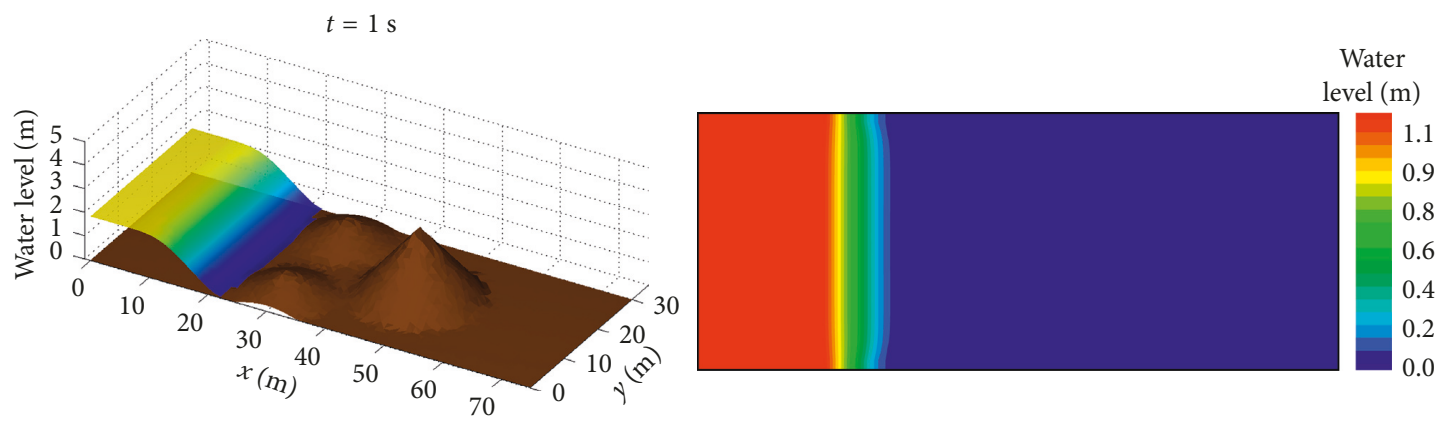

(a)
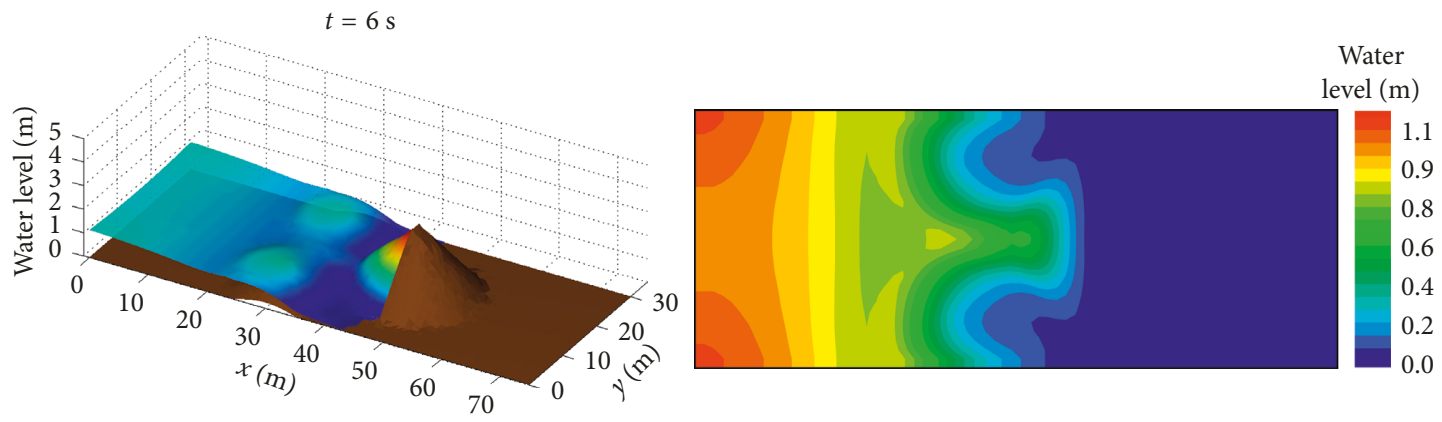

(b)
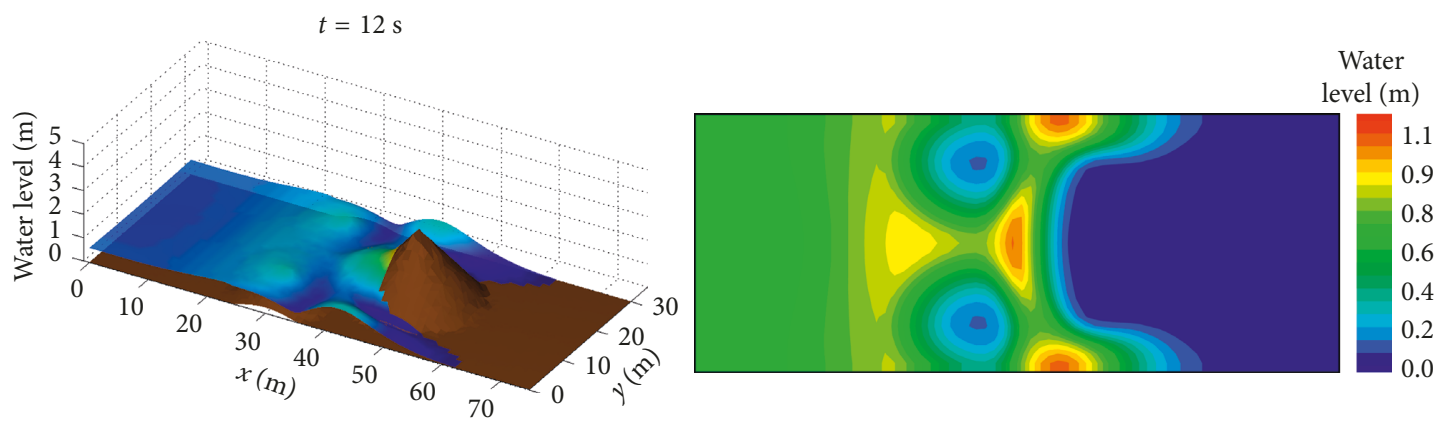

(c)

Figure 9: Continued. 

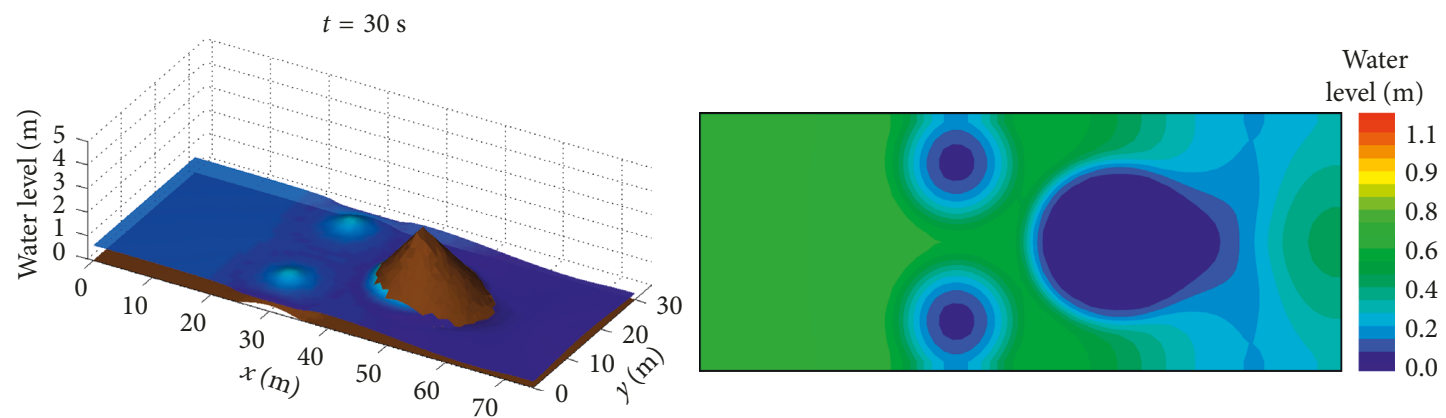

(d)
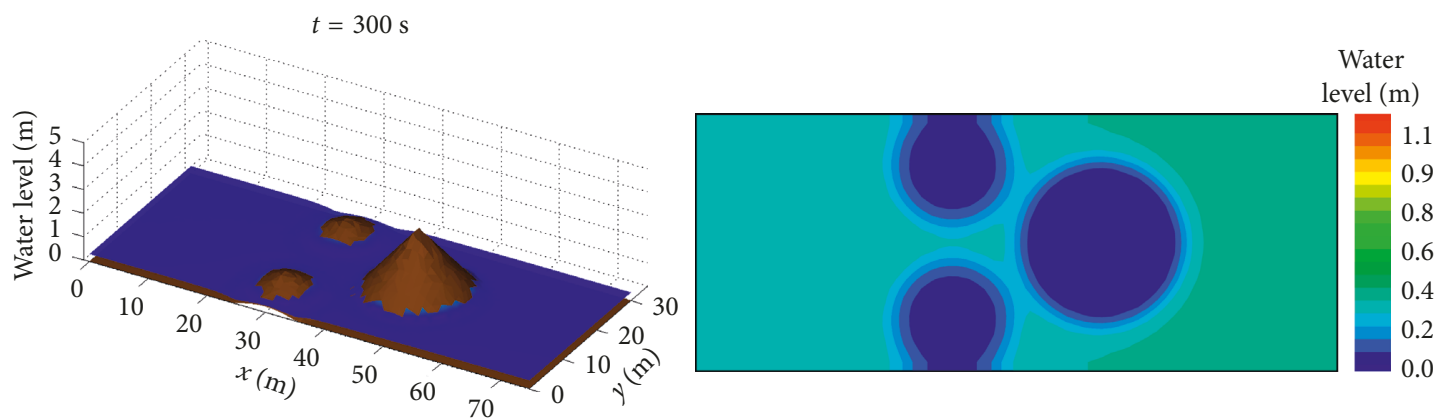

(e)

Figure 9: The profile of water depth and depth contours of the dam break over three humps at different output times: $t=1 \mathrm{~s}, t=6 \mathrm{~s}, t=12 \mathrm{~s}$, $t=30 \mathrm{~s}$, and $t=300 \mathrm{~s}$.

The overall flow pattern for this example is preserved with no spurious oscillations appearing in the results by the RBF meshless method. Obviously, the obtained results verify the stability and the shock-capturing properties of the proposed meshless method. The RBF meshless method performs well for this test problem since it does not diffuse the moving fronts, and no spurious oscillations have been observed when the water flows over the humps.

\section{Conclusions}

This paper has investigated a simple RBF meshless method for numerical simulation of dam break flows by solving the shallow water equations including bed friction and nonuniform bed elevation terms. The space derivative has been approximated by radial basis functions on global collocation points. An explicit third-order Runge-Kutta scheme was used for time integration. The RBF meshless method has been found to be flexible and straightforward to implement. The method is particularly attractive for the shallow water equations in comparison with classical mesh-based methods because it is inherently mesh-free and requires no special treatment of the wet-dry interface. The method was tested against several theoretical dam break problems, including a dam break with a nonsymmetric breach, a circular dam break, and a dam break on an irregular bed involving the wet/dry interface. The numerical model gives promising predictions in comparison with previously references and published results.

\section{Conflicts of Interest}

The author declares that there are no conflicts of interest regarding the publication of this paper.

\section{References}

[1] T. Belytschko, Y. Krongauz, D. Organ, M. Fleming, and P. Krysl, "Meshless methods: an overview and recent developments," Computer Methods in Applied Mechanics and Engineering, vol. 139, no. 1-4, pp. 3-47, 1996.

[2] G. Liu, Mesh Free Methods: Moving Beyond the Finite Element Method, CRC Press, Boca Raton, FL, USA, 2002.

[3] V. Nguyen, T. Rabczuk, S. Bordas, and M. Duflot, "Meshless methods: a review and computer implementation aspects," Mathematics and Computers in Simulation, vol. 79, no. 3, pp. 763-813, 2008.

[4] A. Fili, A. Naji, and Y. Duan, "Coupling three-field formulation and meshless mixed Galerkin methods using radial basis functions," Journal of Computational and Applied Mathematics, vol. 234, no. 8, pp. 2456-2468, 2010.

[5] E. J. Kansa, "A scattered data approximation scheme with applications to computational fluid-dynamics-I and II," Computers and Mathematics with Applications, vol. 19, no. 89, pp. 127-161, 1990.

[6] A. Bratsos, "An improved numerical scheme for the sine-Gordon equation in $2+1$ dimensions," International Journal for Numerical Methods in Engineering, vol. 75, no. 7, pp. 787-799, 2008.

[7] Y. Gu and G. Liu, "A boundary point interpolation method for stress analysis of solids," Computational Mechanics, vol. 28, no. 1, pp. 47-54, 2002.

[8] G. Liu, L. Yan, J. Wang, and Y. Gu, "Point interpolation method based on local residual formulation using radial basis functions," Structural Engineering and Mechanics, vol. 14, no. 6, pp. 713-732, 2002.

[9] E. Shivanian, "On the convergence analysis, stability, and implementation of meshless local radial point interpolation on a class of three-dimensional wave equations," International 
Journal for Numerical Methods in Engineering, vol. 105, no. 2, pp. 83-110, 2016.

[10] L. M. J. S. Dinis, R. M. Natal Jorge, and J. Belinha, "Analysis of 3D solids using the natural neighbour radial point interpolation method," Computer Methods in Applied Mechanics and Engineering, vol. 196, no. 13-16, pp. 2009-2028, 2007.

[11] J. Belinha, L. M. J. S. Dinis, and R. M. Natal Jorge, "The natural radial element method," International Journal for Numerical Methods in Engineering, vol. 93, no. 12, pp. 1286-1313, 2013.

[12] A. Fedoseyev, M. Friedman, and E. Kansa, "Improved multiquadric method for elliptic partial differential equations via PDE collocation on the boundary," Computers and Mathematics with Applications, vol. 43, no. 3-5, pp. 491-500, 2002.

[13] A. Cheng, M. Golberg, E. Kansa, and T. Zammito, "Exponential convergence and $\mathrm{H}-\mathrm{c}$ multiquadric collocation method for partial differential equations," Numerical Methods for Partial Differential Equations, vol. 19, no. 5, pp. 571-594, 2003.

[14] C. Shu, H. Ding, H. Chen, and T. Wang, "An upwind local RBF-DQ method for simulation of inviscid compressible flows," Computer Methods in Applied Mechanics and Engineering, vol. 194, no. 18-20, pp. 2001-2017, 2005.

[15] H. Ding, C. Shu, K. Yeo, and Z. Lu, "Simulation of natural convection in eccentric annuli between a square outer cylinder and a circular inner cylinder using local MQ-DQ method," Numerical Heat Transfer, Part A: Applications, vol. 47, no. 3, pp. 291-313, 2005.

[16] S. Soleimani, D. Ganji, E. Ghasemi, M. Jalaal, and H. Bararnia, "Meshless local RBF-DQ for 2-D heat conduction: a comparative study," Thermal Science, vol. 15, no. 1, pp. 117-121, 2011.

[17] H. Ding, C. Shu, K. Yeo, and D. Xu, "Numerical computation of three-dimensional incompressible viscous flows in the primitive variable form by local multiquadric differential quadrature method," Computer Methods in Applied Mechanics and Engineering, vol. 195, no. 7-8, pp. 516-533, 2006.

[18] A. Khoshfetrat and M. Abedini, "Numerical modeling of long waves in shallow water using LRBF-DQ and hybrid DQ// LRBF-DQ," Ocean Model, vol. 65, pp. 1-10, 2013.

[19] M. Antuono, L. Soldini, and M. Brocchini, "On the role of the Chezy frictional term near the shoreline," Theoretical and Computational Fluid Dynamics, vol. 26, no. 1-4, pp. 105-116, 2012.

[20] E. Kansa, "Exact explicit time integration of hyperbolic partial differential equations with mesh free radial basis functions," Engineering Analysis with Boundary Elements, vol. 31, no. 7, pp. 577-585, 2007.

[21] G. Kesserwani and Q. Liang, "Locally limited and fully conserved RKDG shallow water solutions with wetting and drying," Journal of Scientific Computing, vol. 50, no. 1, pp. 120-144, 2012.

[22] P. Ullrich, C. Jablonowski, and B. van Leer, "High-order finite-volume methods for the shallow water equations on the sphere," Journal of Computational Physics, vol. 229, no. 17, pp. 6104-6134, 2010.

[23] D. Stevens and H. Power, "The radial basis function finite collocation approach for capturing sharp fronts in time dependent advection problems," Journal of Computational Physics, vol. 298, pp. 423-445, 2015.

[24] E. Chaabelasri, A. Amahmouj, M. Jeyar, A. G. L. Borthwick, N. Salhi, and I. Elmahi, "Numerical survey of contaminant transport and self cleansing of water in Nador Lagoon, Morocco," Modelling and Simulation in Engineering, vol. 2014, Article ID 179504, 8 pages, 2014.

[25] S. Sarra, "Integrated multiquadric radial basis function approximation methods," Computers and Mathematics with Applications, vol. 51, no. 8, pp. 1283-1296, 2006.
[26] F. Benkhaldoun, A. Halassi, D. Ouazar, M. Seaid, and A. Taik, "A stabilized meshless method for time-dependent convectiondominated flow problems," Mathematics and Computers in Simulation, vol. 137, pp. 159-176, 2017.

[27] F. Boushaba, E. M. Chaabelasri, N. Salhi, I. Elmahi, F. Benkhaldoun, and A. G. L. Borthwick, "A comparative study of finite volume and finite element on some transcritical free surface flow problems," International Journal of Computational Methods, vol. 5, no. 3, pp. 413-431, 2008.

[28] X. Xia, Q. Liang, M. Pastor, W. Zou, and Y. Zhuang, "Balancing the source terms in a SPH model for solving the shallow water equations," Advances in Water Resources, vol. 59, pp. 25-38, 2013.

[29] S. J. Jackson, D. Stevens, D. Giddings, and H. Power, "An adaptive RBF finite collocation approach to track transport processes across moving fronts," Computers and Mathematics with Applications, vol. 71, no. 1, pp. 278-300, 2016.

[30] D. Liang, B. Lin, and R. A. Falconer, "Simulation of rapidly varying flow using an efficient TVD-MacCormack scheme," International Journal for Numerical Methods in Fluids, vol. 53, no. 5, pp. 811-826, 2007.

[31] H. Shirkhania, A. Mohammadian, O. Seidou, and A. Kurganov, "A well balanced positivity preserving central upwind scheme for shallow water equations on unstructured quadrilateral grids," Computers and Fluids, vol. 126, pp. 25-40, 2016.

[32] I. K. Nikolos and A. I. Delis, "An unstructured node-centered finite volume scheme for shallow water flows with wet/dry fronts over complex topography," Computer Methods in Applied Mechanics and Engineering, vol. 198, no. 4748, pp. 3723-50, 2009.

[33] C. K. Chou, C. P. Sun, D. L. Young, J. Sladek, and V. Sladek, "Extrapolated local radial basis function collocation method for shallow water problems," Engineering Analysis with Boundary Elements, vol. 50, pp. 275-290, 2015.

[34] A. Baghlani, "Simulation of dam-break problem by a robust flux-vector splitting approach in Cartesian grid," Scientia Iranica A, vol. 18, no. 5, pp. 1061-1068, 2011.

[35] A. Canestrelli, M. Dumbser, A. Siviglia, and E. F. Toro, "Wellbalanced high-order centered schemes on unstructured meshes for shallow water equations with fixed and mobile bed," Advances in Water Resources, vol. 33, no. 2010, pp. 291-303, 2010.

[36] M. Antuono and M. Brocchini, "Analysis of the nonlinear shallow water equations over non-planar topography," Studies in Applied Mathematics, vol. 124, no. 1, pp. 85-103, 2010.

[37] M. Kawahara and T. Umetsu, "Finite element method for moving boundary problems in river flow," International Journal for Numerical Methods in Fluids, vol. 6, no. 6, pp. 365-86, 1986. 


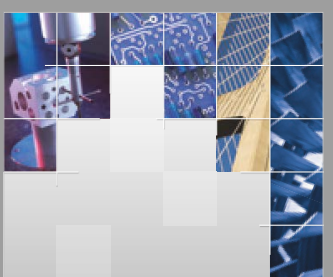

\section{Enfincering}
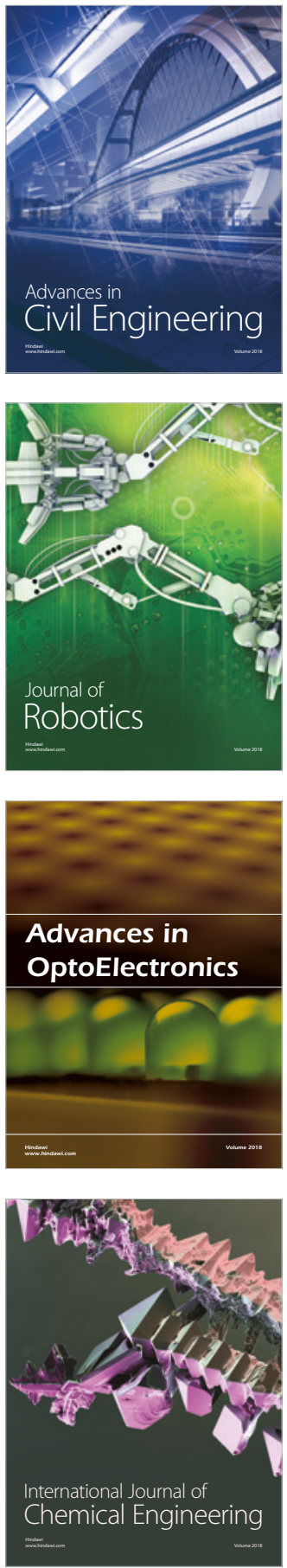

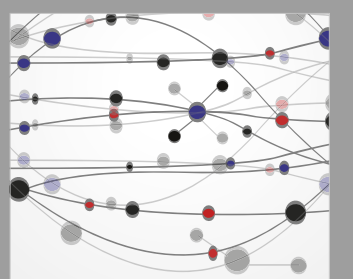

\section{Rotating \\ Machinery}

The Scientific World Journal

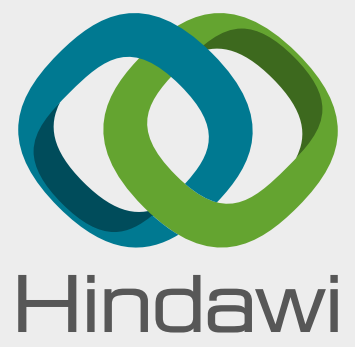

Submit your manuscripts at

www.hindawi.com
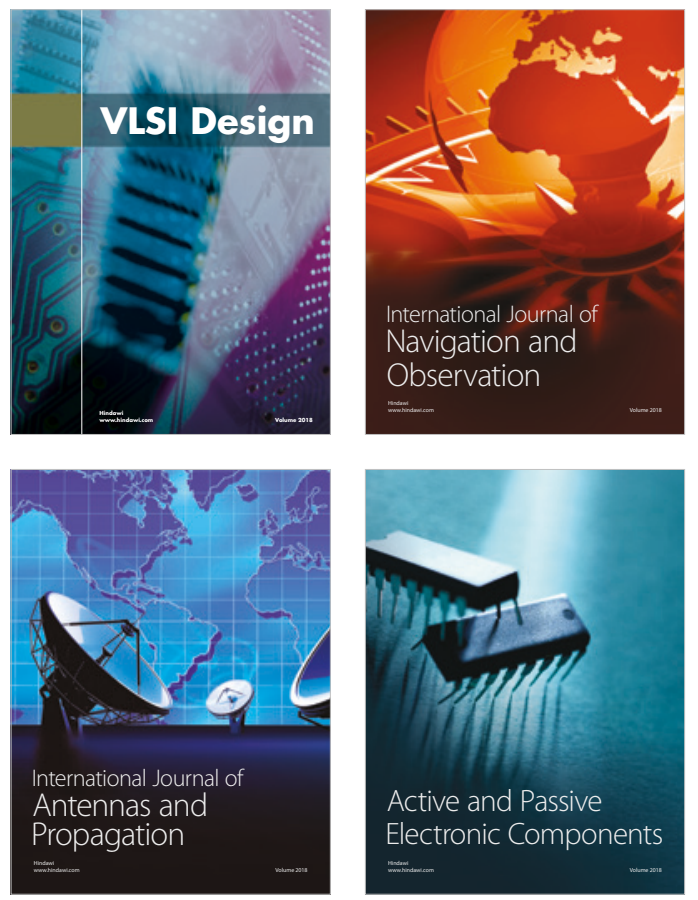
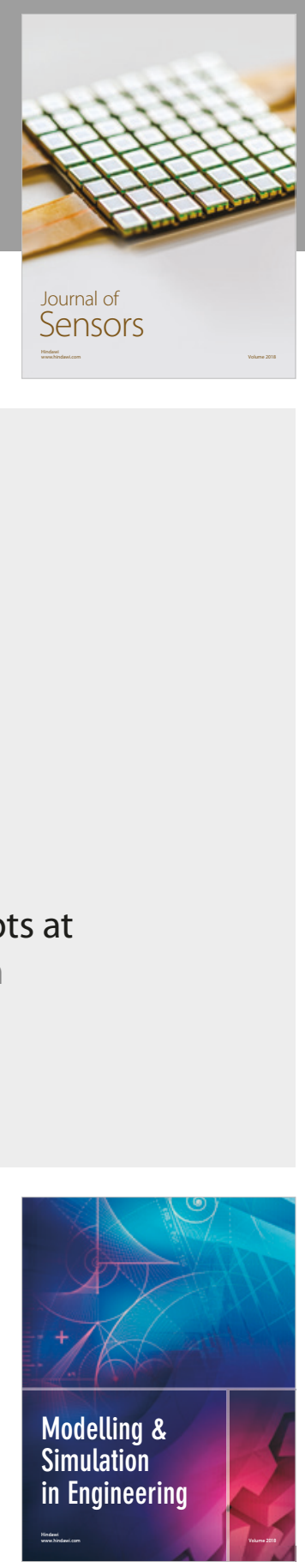

\section{Advances \\ Multimedia}
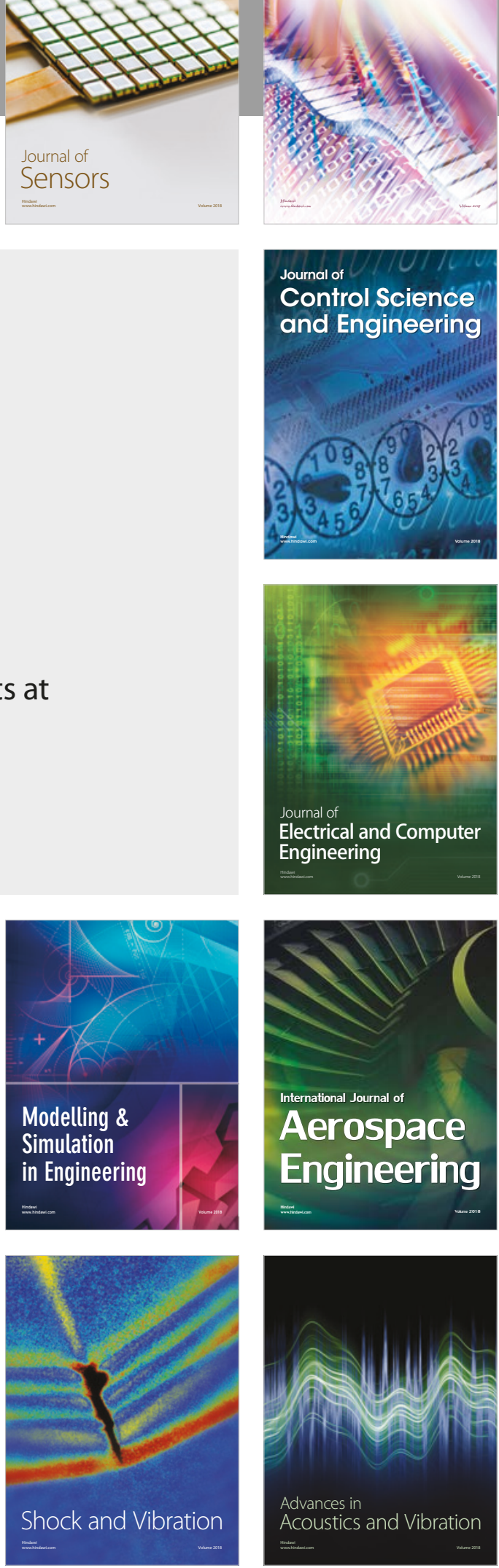